\title{
Histopathological Analysis of Ligamentum Flavum in Lumbar Spinal Stenosis and Disc Herniation
}

\author{
Idiris Altun, Kasım Zafer Yüksel \\ Department of Neurosurgery, Kahramanmaras Sutcu Imam University Medical Faculty, Kahramanmaras, Turkey
}

Study Design: Histopathological analyses were performed in ligamentum flavum (LF) hypertrophy patients with lumbar spinal stenosis (LSS) and lumbar disc herniation (LDH).

Purpose: The aim of the present study was to evaluate histopathological changes in LF patients with LSS and LDH.

Overview of Literature: LSS is the most common spinal disorder in elderly patients. This condition causes lower back and leg pain and paresis, and occurs as a result of degenerative changes in the lumbar spine, including bulging of the intervertebral discs, bony proliferation of the facet joints, and LF thickening; among these, LF thickening is considered a major contributor to the development of LSS.

Methods: A total of 71 patients operated with the surgical indications of LSS and LDH were included. LF samples were obtained from 31 patients who underwent decompressive laminectomy for symptomatic degenerative LSS (stenotic group) and from 40 patients who underwent lumbar discectomy for LDH (discectomy group). LF materials were examined histopathologically, and other specimens were examined for collagen content, elastic fiber number and array, and presence of calcification.

Results: The stenotic and discectomy groups did not differ with regard to mean collagen concentration or mean elastic fiber number $(p=0.430$ and $p=0.457$, respectively). Mean elastic fiber alignment was $2.36 \pm 0.99$ in the stenotic group and $1.38 \pm 0.54$ in the discectomy group $(p<0.001)$. Mean calcification was $0.39 \pm 0.50$ in the stenotic group, whereas calcification was not detected $(0.00 \pm 0.00)$ in the discectomy group; a statistically significant difference was detected $(p<0.001)$ between groups.

Conclusions: LF hypertrophy in spinal stenosis may occur as a result of elastic fiber misalignment along with the development of calcification over time. Further studies determining the pathogenesis of LSS are needed.

Keywords: Spinal stenosis; Ligamentum flavum; Hypertrophy; Pathology

\section{Introduction}

The most common spinal disorder in elderly patients is lumbar spinal stenosis (LSS), which causes lower back and leg pain and paresis. LSS occurs as a result of degenerative changes in the lumbar spine, including bulging of the intervertebral discs, bony proliferation of the facet joints, and ligamentum flavum (LF) thickening [1,2]; among these, LF thickening is considered a major contributor to the development of LSS [3].

Changes in LF related to degeneration are secondary to either the aging process or mechanical instability. Previous studies have indicated that hypertrophied LF shows elastic fiber loss and increased collagen content $[4,5]$. Yoshida et al. [3] identified type-II collagen in hypertrophied LF. Sairyo et al. [6] reported that inflammatory cytokines

Received Jun 10, 2016; Revised Jun 29, 2016; Accepted Jul 2, 2016

Corresponding author: Idiris Altun

Department of Neurosurgery, Kahramanmaras Sutcu Imam University Medical Faculty, Kahramanmaras 46050, Turkey

Tel: +90-3442803434, Fax: +90-3442803409, E-mail: idrisaltun46@hotmail.com 
are expressed in LF and that repetitive inflammation could cause scar accumulation, which would gradually lead to an increase in thickness. Although some growth factors, such as cytokines, matrix metalloproteinases, and tissue inhibitors of matrix metalloproteinases, may play important roles in the pathogenesis of hypertrophy of LF, its mechanism is unclear [7].

In the present study, we aimed to compare histopathological changes in LF in patients with LSS and lumbar disc herniation $(\mathrm{LDH})$.

\section{Materials and Methods}

\section{Study design}

This study was conducted in accordance with the principles of the Helsinki Declaration and approved by the local Institutional Review Board (197/11.2014). Written informed consent was obtained from all participants.

This study included 71 patients who were admitted to the Department of Neurosurgery in our tertiary center between January 2014 and March 2015 and were operated with surgical indications of LSS and LDH. Inclusion criteria were as follows: age $\geq 18$ years, radiating pain and/or paresis below the knee level, and LDH at the corresponding level and side verified by magnetic resonance imaging (MRI) scan. Patients with a history of osteoporosis, immunosuppression, chronic corticosteroid use, intravenous drug use, fever of unknown origin, history of cancer, unexplained weight loss, or progressive/disabling symptoms associated with focal neurological deficits were excluded from the study.

LF samples were obtained from the 31 patients who underwent decompressive laminectomy for symptomatic degenerative LSS (stenotic group) and from the 40 patients who underwent lumbar discectomy for LDH (discectomy group).

LDH or LSS was diagnosed if there were significant MRI findings indicative of these conditions and if clinical manifestations were thought to be compatible with the MRI results. There was no calcification of LF according to preoperative computed tomography scans.

\section{Outcome parameters}

LF materials harvested from patients during surgery were subjected to histopathological analyses. LF materials were kept in a solution containing $10 \%$ formalin for 24 hours. Subsequent to preparation with ethanol and xylene, tissue was placed in paraffin and sections was made at thickness of $4 \mu \mathrm{m}$. Tissue preparations were stained with hematoxylin and eosin and orcein, and were evaluated by two pathologists who were blinded to the nature of the groups. Specimens were examined with regard to hemosiderin and amyloid deposition, collagen content, and elastic fiber number and array. The presence of calcification was scored as follows: 0 , absent; 1 , mild; 2 , moderate; and 3, severe (Fig. 1) $[6,8]$.

\section{Statistical analysis}

Data were analyzed using the IBM Statistical Package for Social Sciences ver. 17 (SPSS Inc., Chicago, IL, USA). Parametric tests were applied to data of normal distribution and nonparametric tests were applied to data of questionably normal distribution. Continuous data were presented as mean \pm standard deviation or median (minimum-maximum), as appropriate. All differences associated with a chance probability of $\leq 0.05$ were considered statistically significant.

\section{Results}

Seventy-one patients ( 35 males, 36 females) met the eligibility criteria for the study. Of these patients whose charts were reviewed, the mean age was 40.74 years (range, 26-53 years). The stenotic group included 31 patients (15 males, 16 females) with a mean age of 41.16 years, whereas the discectomy group included 40 patients (20 males, 20 females) with a mean age of 40.46 years. Groups did not differ from each other regarding age and gender ( $p=0.437$ and $p=0.242$, respectively).

The mean collagen concentration was $2.23 \pm 1.06 \mathrm{mg} /$ $\mathrm{mL}$ in the stenotic group and $2.18 \pm 0.96 \mathrm{mg} / \mathrm{mL}$ in the discectomy group ( $p=0.430$ ). The mean elastic fiber number was $2.16 \pm 1.03$ in the stenotic group and $1.71 \pm 0.79$ in the discectomy group $(p=0.457)$. The mean elastic fiber alignment was $2.36 \pm 0.99$ in the stenotic group and $1.38 \pm 0.54$ in the discectomy group $(p<0.001)$. The mean calcification was $0.39 \pm 0.50$ in the stenotic group, whereas calcification was not detected $(0.00 \pm 0.00)$ in the discectomy group; the difference was statistically significant $(p<0.001)$ (Table 1$)$.

Hemosiderin and amyloid deposition were not observed in any patients. 

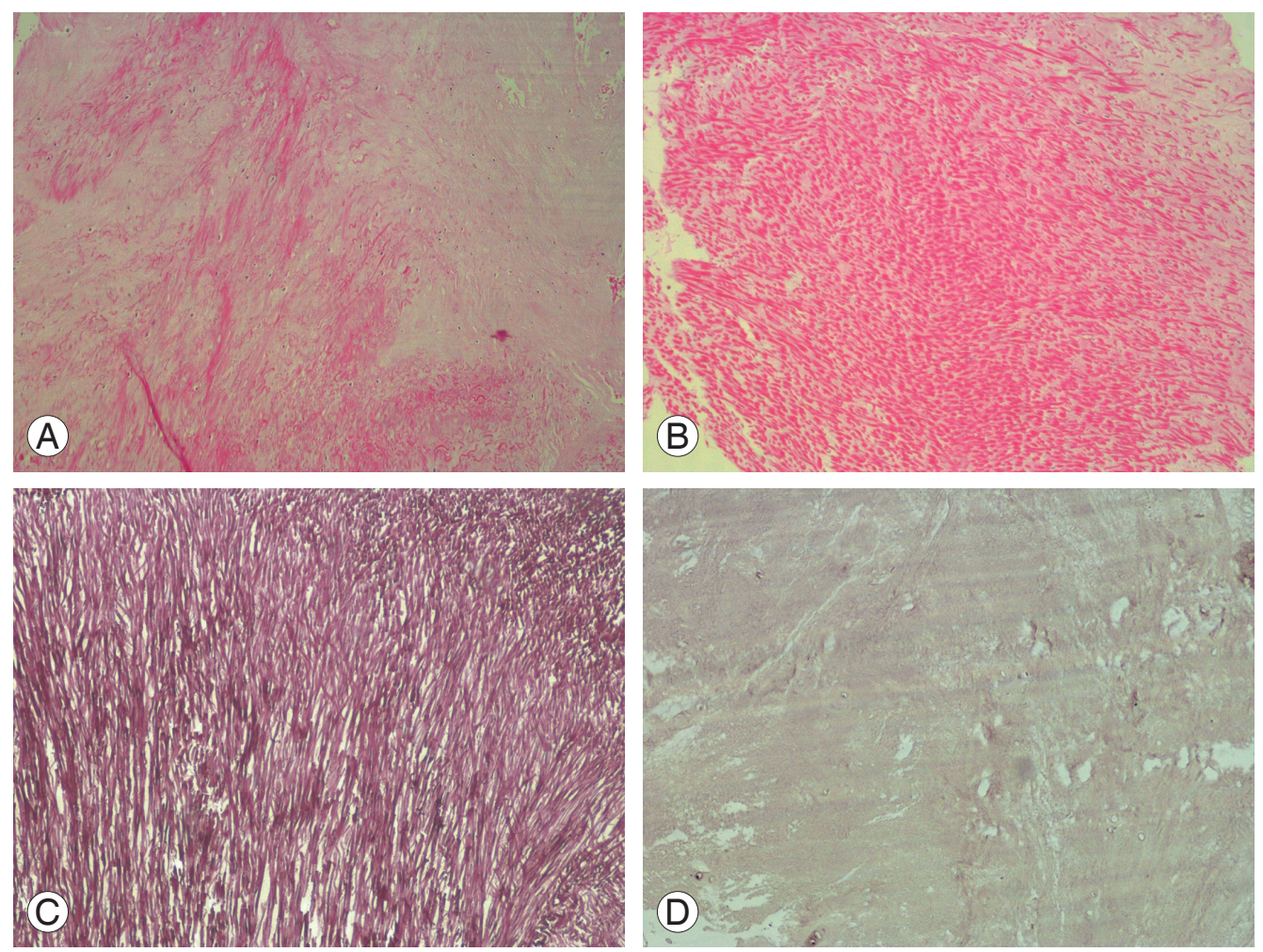

Fig. 1. (A) Collagen-rich tissue (H\&E, $\times 100)$. (B) Collagen-poor, elastic fiber-rich tissue $(H \& E, \times 100)$. (C) Collagenpoor, elastic fiber-normal tissue (orcein, $\times 100)$. (D) Collagen-rich, elastic fiber-poor tissue (orcein, $\times 100$ ).

Table 1. Histopathological changes of ligamentum flavum in patients with lumbar spinal stenosis and lumbar disc herniation

\begin{tabular}{lccc} 
Variable & Stenotic group $(\mathrm{n}=31)$ & Discectomy group $(\mathrm{n}=40)$ & $p$-value \\
Collagen concentration $(\mathrm{mg} / \mathrm{mL})$ & $2.23 \pm 1.06$ & $2.18 \pm 0.96$ & 0.430 \\
No. of elastic fibers & $2.16 \pm 1.03$ & $1.71 \pm 0.79$ & 0.457 \\
Elastic fiber alignment & $2.36 \pm 0.99$ & $1.38 \pm 0.54$ & $<0.001$ \\
Calcification & $0.39 \pm 0.50$ & $0.00 \pm 0.00$ & $<0.001$ \\
\hline
\end{tabular}

\section{Discussion}

Increased recognition of the nature of hypertrophied LF has highlighted the importance of understanding its pathogenesis. Herein, we aimed to compare the histopathological changes in LF in patients with LSS and LDH. We found that statistically significant differences in mean elastic fiber alignment and mean calcification levels were observed between the two groups.

LF covers the posterior and lateral walls of the spinal canal. As LF thickens, it compresses the nerve roots of the cauda equina [6], and surgical removal of the thickened LF can help treat LSS [9]. The normal LF is a well-defined elastic structure containing $80 \%$ elastic fibers and $20 \%$ collagen fibers. Upon hypertrophy, LF shows elastic fiber loss and increased collagen fibers, which result in fibrosis $[5,10]$. Loss of elasticity may cause LF to fold into the spinal canal, which may further narrow of the canal.

Previous studies have provided important data regarding the pathogenesis of LF hypertrophy $[4,6,10]$. In these studies, collagen replacement, alterations in the structure and array of elastic fibers and collagen/elastin ratio and calcification were separately evaluated; however, none of these studies evaluated all of these parameters in combination.

LF contains the purest form of elastic tissue among ligaments; these elastic fibers decrease with age and are replaced by collagen fibers [4-6]. In a study performed in 
fetuses, young patients, and old patients, reductions in LF elasticity were associated with a decrease in the presence of elastic fibers and an increase in the presence of collagen fibers [4]. The authors suggested that LF hypertrophy could result from increased elasticity and abnormal collagen structure. It has been also reported that LSS patients have elastic fiber content reduction and misalignment; however, these results were not seen in $\mathrm{LDH}$ patients. In the present study, although a reduction in elastic fibers and an increase in collagen fibers were observed, no statistically significant differences were detected between the groups; however, we did find a significant difference in elastic fiber misalignment between the groups.

The main limitation of this study was the relatively small sample size. Second, we used control specimens from patients with disc herniations because we were unable to collect normal LF samples. Third, the mean age of patients in the stenotic group did not differ from that of the discectomy group, which could explain why we did not find statistically significant differences between the two groups in elastic fiber number and in collagen concentrations. Since subjects in both the groups were very young, it is possible that LSS patients at age 50 years may differ from elderly LSS patients with regard to symptom presentation. Because of these limitations, our results should be interpreted with caution. In addition, it is important to note our analysis did not focus on the mechanism of the development of spinal stenosis.

\section{Conclusions}

LF hypertrophy in spinal stenosis may occur as a result of elastic fiber misalignment along with development of calcification over time. Further studies determining the pathogenesis of LSS are needed.

\section{Conflict of Interest}

No potential conflict of interest relevant to this article was reported.

\section{Acknowledgments}

The author would like to thank Harun Çıralık, MD, for assistance with the histopathological evaluation.

\section{References}

1. Nakatani T, Marui T, Hitora T, Doita M, Nishida K, Kurosaka M. Mechanical stretching force promotes collagen synthesis by cultured cells from human ligamentum flavum via transforming growth factorbeta1. J Orthop Res 2002;20:1380-6.

2. Hansson T, Suzuki N, Hebelka H, Gaulitz A. The narrowing of the lumbar spinal canal during loaded MRI: the effects of the disc and ligamentum flavum. Eur Spine J 2009;18:679-86.

3. Yoshida M, Shima K, Taniguchi Y, Tamaki T, Tanaka T. Hypertrophied ligamentum flavum in lumbar spinal canal stenosis: pathogenesis and morphologic and immunohistochemical observation. Spine (Phila Pa 1976) 1992;17:1353-60.

4. Kosaka H, Sairyo K, Biyani A, et al. Pathomechanism of loss of elasticity and hypertrophy of lumbar ligamentum flavum in elderly patients with lumbar spinal canal stenosis. Spine (Phila Pa 1976) 2007;32: 2805-11.

5. Zhong ZM, Zha DS, Xiao WD, et al. Hypertrophy of ligamentum flavum in lumbar spine stenosis associated with the increased expression of connective tissue growth factor. J Orthop Res 2011;29:1592-7.

6. Sairyo K, Biyani A, Goel VK, et al. Lumbar ligamentum flavum hypertrophy is due to accumulation of inflammation-related scar tissue. Spine (Phila $\mathrm{Pa}$ 1976) 2007;32:E340-7.

7. Park JB, Chang H, Lee JK. Quantitative analysis of transforming growth factor-beta 1 in ligamentum flavum of lumbar spinal stenosis and disc herniation. Spine (Phila Pa 1976) 2001;26:E492-5.

8. Okuda T, Baba I, Fujimoto Y, et al. The pathology of ligamentum flavum in degenerative lumbar disease. Spine (Phila Pa 1976) 2004;29:1689-97.

9. Zhang Y, Chen J, Zhong ZM, Yang D, Zhu Q. Is platelet-derived growth factor-BB expression proportional to fibrosis in the hypertrophied lumber ligamentum flavum? Spine (Phila Pa 1976) 2010;35:E1479-86.

10. Altinkaya N, Yildirim T, Demir S, Alkan O, Sarica FB. Factors associated with the thickness of the ligamentum flavum: is ligamentum flavum thickening due to hypertrophy or buckling? Spine (Phila Pa 1976) 2011;36:E1093-7. 